\title{
Risk Factors for Childhood Overweight and Obesity in Ukraine and Germany
}

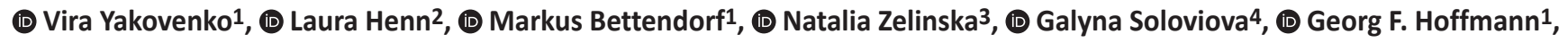 \\ (D) Juergen Grulich-Henn'1
}

${ }_{1}^{1}$ Ruprecht-Karls-University Heidelberg, University Children's Hospital, Department of General Pediatrics, Heidelberg, Germany

2Otto-von-Guericke University Magdeburg, Institute of Psychology, Magdeburg, Germany

${ }^{3}$ Ukrainian Center of Endocrine Surgery and Transplantation of Endocrine Organs and Tissues, Kiev, Ukraine

4 Ukrainian Children Specialized Hospital "OHMATDIT", Kiev, Ukraine

\begin{abstract}
What is already known on this topic?
Obesity in children and adolescents has become an increasing and widely-distributed health problem, especially in industrialized countries. Risk factors include both genetic predisposition and socioeconomic factors. Only a few studies have compared the distribution of socioeconomic risk factors for childhood obesity across different countries.
\end{abstract}

\section{What this study adds?}

The current study is the first one to analyze the distribution of risk factors for obesity in Ukraine and Germany. Similar risk factors for obesity were observed in both countries although the prevalence of these risk factors varied between the two populations.

\begin{abstract}
Objective: The prevalence of overweight and obesity in childhood and adolescence are rapidly increasing and influenced by genetic, familial, environmental, socioeconomic and cultural factors. The aim of the study was to compare risk factors for childhood obesity in Ukraine (UA) and Germany (DE) using comparable investigative tools.

Methods: Two groups of children, aged 8 to 18 years, from DE (93 children) and UA (95 children) were divided into overweight and obese groups. Anthropometric data and detailed medical history were collected.

Results: Risk factors in pregnancy (prematurity, weight gain $>20 \mathrm{~kg}$, early contractions) were equally frequent in both groups. Positive correlations of body mass index (BMI)-standard deviation score (SDS) between children and mothers were noted. The proportion of family members with diabetes mellitus was lower in the UA group. Obesity was more frequent at one year of age in DE children. The DE group also became overweight at an earlier age and remained overweight over a longer period of time compared to UA. The mean BMI-SDS of obese children was lower in the UA group. In both groups waist circumference to height ratio was $>0.5$, indicating presence of a cardiometabolic risk factor. About half of the patients in both groups had blood pressure values exceeding the 95 th percentile.

Conclusion: Similar risk factors for obesity were observed among two groups of children in UA and DE. Differences were observed regarding the prevalence of specific risk factors for childhood obesity. Population-specific distribution of risk factors needs to be considered in order to optimize prevention and treatment strategies.
\end{abstract}

Keywords: Obesity, risk factors, childhood, adolescent, overweight

\section{Introduction}

Obesity in children and adolescents has become an increasing and widespread health problem, especially in industrialized countries. According to the World Health Organization (WHO) more than 41 million children under the age of five suffered from overweight or obesity in 2016 (1). In Germany (DE), according to two large national studies (KIGGS and Crescnet), 15-17\% of children aged three to 17 years were overweight and 6.2-7.6\% were obese (2). In Ukraine (UA), the incidence of childhood obesity until
Address for Correspondence: Juergen Grulich-Henn MD, Ruprecht-Karls-University Heidelberg, University Children's Hospital, Department of General Pediatrics, Heidelberg, Germany

Phone: +496221564002 E-mail: juergen.grulich-henn@med.uni-heidelberg.de

ORCID: orcid.org/0000-0003-2118-8615

${ }^{\circ}$ Copyright 2019 by Turkish Pediatric Endocrinology and Diabetes Society

The Journal of Clinical Research in Pediatric Endocrinology published by Galenos Publishing House.
Conflict of interest: None declared Received: 17.06 .2018 Accepted: 05.01.2019 
recently was much lower but has shown a significant rise over the last decade, increasing from $0.083 \%$ among age groups $0-18$ years in 2003, to $1.23 \%$ in 2009 and 1.34 in $2016(3,4)$

Numerous risk factors for childhood obesity have been discussed in the literature. Parental overweight is considered to be a particularly important indicator for overweight and obesity among their children (5). Further risk factors include characteristics of the maternal medical history during pregnancy and the perinatal period. Thus, excessive maternal weight gain during pregnancy and maternal smoking during pregnancy contribute to the development of obesity and its incidence (6). In addition, high birth weight constitutes a significant risk factor for the development of childhood obesity (7). Moreover, children with rapid weight gain during the first four to six months of life have been shown to have an increased risk of developing obesity by the age of seven years. Breastfeeding over a period of at least six months reduces the risk of obesity by $40 \%$ (8). Children who have become overweight by the age of six years often remain overweight at age 14 years (9).

Since obviously both genetic and socioeconomic factors have an influence on the development of childhood obesity, we investigated similarities and differences regarding these risk factors in two cohorts from two different European countries (UA and DE).

\section{Methods}

The study was conducted at two university children hospital outpatient centers, in Simferopol, UA (2010-2011) and in Heidelberg, DE (2012-2013). At both centers WHO standards were used for definition of overweight and obesity. Inclusion criteria were: age 8-18 years, BMI 85th-95th percentile (overweight) and $>95^{\text {th }}$ percentile (obesity), informed consent. Exclusion criteria were: age $<8$ years or $>18$ years, chronic endocrinopathies (diabetes mellitus ect.), genetic disorders, disabled children, chronic inflammatory diseases (e.g. M. Crohn), lack of informed consent. Inclusion and exclusion criteria were identical. Comparable questionnaires for risk factors and for both patient and family medical history were used at both centers.

The study population in the UA consisted of 95 (35 girls and 60 boys) otherwise healthy children aged 10 to 18 [mean \pm standard deviation $(S D)=13.5 \pm 0.4]$ years, divided into an overweight (36 children) and an obesity (59 children) group. The study population in DE consisted of 93 (46 girl, 47 boys) otherwise healthy children aged 8 to 18 years (mean $\pm \mathrm{SD}=12.5 \pm 2.9$ ) years divided into an overweight ( 24 children) and an obesity (69 children) group.
The populations were comparable according to sex, Tanner stages and age and did not show any statistically significant difference. Physical examination in outpatient departments included measurement of height, weight (in underwear), waist and hip circumference, blood pressure and examination of the skin for acanthosis nigricans. BMI-SDS, waist/hip circumference ratio (WHR), and waist circumference/height ratio (WHtR) were calculated. Standardized patient history included pregnancy history (maternal obesity, weight gain of more than $20 \mathrm{~kg}$, arterial hypertension, premature (before $37^{\text {th }}$ gestational week) or postmature (after $42^{\text {nd }}$ gestational week) delivery, perinatal asphyxia, duration of breastfeeding and family history (overweight or obesity, diabetes mellitus, arterial hypertension in first degree relatives). All risk factors were recorded with regard to their presence or absence. The children's history of weight gain was also evaluated (reported by parents and according to medical reports).

The study was approved by the Ethics Committee of the Medical Faculty of the University of Heidelberg (approval number: S-337/2013, approval date: 22/07/2013). Written consent was taken from the parents at the beginning of the study in accordance with the Declaration of Helsinki.

\section{Statistical Analysis}

Statistical analysis was performed using Statistical Package for the Social Sciences (SPSS), version 20.0.0 for Windows (SPSS Inc., Chicago, IL., USA). As part of the descriptive analysis, sample size, arithmetic mean, median, maximum (max) and minimum (min), and SD were determined. To test the variables for normality of the distribution, the Kolmogorov-Smirnov test with an error probability of 0.05 was used. The t-test and the Mann-Whitney $U$ test were used to test for significant differences between groups. Gender differences in categorical variables were tested with the chi-square test. A statistical parameter of $p<0.05$ was considered significant.

\section{Results}

The risk factors during pregnancy occurred with an approximately equal frequency among the DE and UA populations (Table 1). Premature birth was reported in $8-12 \%$ of all children. In the DE population $21 \%$ of mothers of overweight children and $33 \%$ of mothers of obese children gained more than $20 \mathrm{~kg}$ during pregnancy. In the UA population the incidence of excessive weight gain during pregnancy among mothers of obese children was $25 \%$. Early contractions were reported by $13-17 \%$ (DE) and 20$28 \%$ (UA) of mothers, respectively (Table 1). 
The prevalence of obesity was significantly higher in firstdegree relatives of obese children compared to relatives of overweight children $(p<0.05)$. A highly significant positive correlation was found between the children's and

Table 1. Prenatal and familial risk factors in the overweight and obese groups

\begin{tabular}{|c|c|c|c|c|}
\hline \multirow[b]{2}{*}{ Risk factors } & \multicolumn{2}{|c|}{$\begin{array}{c}\mathrm{DE} \\
\left(\mathrm{n}_{\text {total }}=93\right)\end{array}$} & \multicolumn{2}{|c|}{$\begin{array}{c}\mathrm{UA} \\
\left(\mathrm{n}_{\text {total }}=95\right)\end{array}$} \\
\hline & $\mathrm{n}$ & $\%$ & $\mathrm{n}$ & $\%$ \\
\hline \multicolumn{5}{|l|}{ Overweight group } \\
\hline \multicolumn{5}{|l|}{ During pregnancy } \\
\hline Maternal obesity & 5 & $21 \%$ & 1 & $3 \%$ \\
\hline Arterial hypertension & 2 & $8 \%$ & 1 & $3 \%$ \\
\hline Early contractions & 4 & $17 \%$ & 10 & $28 \%$ \\
\hline Gestational age $<37$ weeks & 2 & $8 \%$ & 4 & $11 \%$ \\
\hline Perinatal asphyxia & 2 & $8 \%$ & 1 & $3 \%$ \\
\hline \multicolumn{5}{|l|}{ Family history } \\
\hline Diabetes mellitus & 14 & $58 \%$ & 6 & $17 \%$ \\
\hline Arterial hypertension & 12 & $50 \%$ & 14 & $39 \%$ \\
\hline Obesity & 14 & $58 \%$ & 14 & $39 \%$ \\
\hline \multicolumn{5}{|l|}{ Obese group } \\
\hline \multicolumn{5}{|l|}{ During pregnancy } \\
\hline Maternal obesity & 22 & $33 \%$ & 15 & $25 \%$ \\
\hline Arterial hypertension & 11 & $17 \%$ & 4 & $7 \%$ \\
\hline Early contractions & 9 & $13 \%$ & 12 & $20 \%$ \\
\hline Gestational age $<37$ weeks & 7 & $10 \%$ & 7 & $12 \%$ \\
\hline Perinatal asphyxia & 5 & $8 \%$ & 2 & $3 \%$ \\
\hline \multicolumn{5}{|l|}{ Family history } \\
\hline Diabetes mellitus & 47 & $68 \%$ & 17 & $29 \%$ \\
\hline Arterial hypertension & 47 & $68 \%$ & 28 & $47 \%$ \\
\hline Obesity & 55 & $80 \%$ & 37 & $63 \%$ \\
\hline
\end{tabular}

Statistical analyses were not suitable in some parameter, due to small mumbers, and therefore this table is presented in a descriptive manner only. DE: Germany, UA: Ukraine

Table 2. Anthropometric data

\begin{tabular}{llllll}
\hline & & \multicolumn{2}{c}{ DE-Group } & \multicolumn{2}{c}{ UA-Group } \\
\cline { 3 - 6 } BMI-SDS & Overweight & 1.44 & 0.20 & 1.42 & 0.19 \\
\cline { 3 - 6 } & Mean & SD & Mean & SD \\
\cline { 3 - 6 } Tanner 1-2 & Overwe & $2.52^{*}$ & 0.55 & $2.35^{*}$ & 0.49 \\
& Obese & 1.41 & 0.21 & 1.52 & 0.11 \\
Tanner 3-5 & Overweight & 1.47 & 0.19 & 1.35 & 0.19 \\
& Obese & $2.62^{* *}$ & 0.63 & $2.19 * *$ & 0.45
\end{tabular}

* $\mathrm{p}<0.05$ between DE and UA.

** $p<0.05$ between Tanner stages

DE: Germany, UA: Ukraine, BMI-SDS: body mass index-standard deviation score, SD: standard deviation the mothers' BMI-SDS $(1.03 \pm 1.26$ for the overweight and $1.96 \pm 1.20$ for the obese group) in the DE population $(r=0.46, p<0.0001)$.

Birth weight $(3295 \pm 474 \mathrm{~g}$ in UA population, $3352 \pm 517$ $\mathrm{g}$ in DE population) and birth length $(50.6 \pm 2.4 \mathrm{~cm}$ in UA population, $51.1 \pm 2.1$ in DE population) as well as their relation to BMI-SDS did not differ among the two groups. In both populations, obese children were more frequently obese at the age of one year $33.3 \%$ in UA population, $27.3 \%$ in DE population) compared to overweight children (14.3\% in UA population, $17.4 \%$ in DE population). On average, the children in the UA population were breastfed for a longer period of time $(6.8 \pm 6.7$ months in the obese, $7.1 \pm 7.2$ months in the overweight group) compared to the children in the DE population $(4.6 \pm 6.2$ months in the obese, $6.1 \pm 7.3$ months in the overweight group). The DE population became overweight significantly earlier and therefore remained overweight over a significantly longer period. The mean duration of obesity in the DE population was $7.6 \pm 4.3$ years ( $\min 1.2$, $\max 18.0$ ), the mean duration of overweight $7.2 \pm 5.0$ years (min 1.1, max 18.0) compared to $5.7 \pm 3.5$ years ( $\min 1.0, \max 13.7$ ) and $4.71 \pm 3.5$ years (min 0.8, max 14.0), respectively in the UA population. In both populations, the duration of the overweight period significantly influenced the BMI-SDS. The mean BMI-SDS of obese children was lower in the UA population than in those in the DE population [BMI-SDS (UA) $2.31 \pm 0.49$ vs BMISDS (DE) $2.52 \pm 0.55(p<0.05)]$. There were no significant differences in BMI-SDS in overweight children. In both populations, the BMI-SDS was significantly influenced by the Tanner stage $(p<0.05)$ (Table 2).

There were significant differences between the DE and UA populations regarding WHR and WHtR $(p<0.05)$

Table 3. Risk factors for metabolic syndrome

\begin{tabular}{|c|c|c|c|c|c|}
\hline \multirow{2}{*}{ Prevalence } & & \multicolumn{2}{|c|}{ DE-Group } & \multicolumn{2}{|c|}{ UA-Group } \\
\hline & & Mean & SD & Mean & SD \\
\hline \multirow{2}{*}{$\begin{array}{l}\text { Waist/hip } \\
\text { circumference } \\
\text { ratio }\end{array}$} & Overweight & $1.09 *$ & \pm 0.13 & $0.86^{*}$ & \pm 0.09 \\
\hline & Obese & $1.11^{*}$ & \pm 0.10 & $0.84^{*}$ & \pm 0.08 \\
\hline \multirow{3}{*}{$\begin{array}{l}\text { Waist } \\
\text { circumference/ } \\
\text { height ratio }\end{array}$} & Overweight & 0.51 & \pm 0.04 & 0.48 & \pm 0.03 \\
\hline & Obese & 0.58 & \pm 0.07 & 0.55 & \pm 0.05 \\
\hline & & $\mathrm{n}$ & $\%$ & $\mathrm{n}$ & $\%$ \\
\hline \multirow{2}{*}{$\begin{array}{l}\text { Blood pressure } \\
>95^{\text {th }} \\
\text { percentile }\end{array}$} & Obese & 37 & $54 \%$ & 26 & $22 \%$ \\
\hline & Overweight & 8 & $33 \%$ & 12 & $33 \%$ \\
\hline \multirow{2}{*}{$\begin{array}{l}\text { Acanthosis } \\
\text { nigricans }\end{array}$} & Obese & 36 & $52 \%$ & 13 & $22 \%$ \\
\hline & Overweight & 2 & $8 \%$ & 5 & $14 \%$ \\
\hline
\end{tabular}


(Table 3). The children in the UA population showed no significant differences in the WHR according to sex or age compared to the DE population. In the DE population the WHtR was significantly influenced by gender $(p<0.05)$. In both populations children showed central trunk obesity (predominantly the girls in the UA population). In obese children the WHtR exceeded 0.5. In the DE population $54 \%$ of obese patients had blood pressure values above the $95^{\text {th }}$ percentile compared to $22 \%$ in the UA population (Table 3). Acanthosis nigricans was observed twice as often among patients in the DE population. In both populations, acanthosis nigricans was observed significantly more frequently in obese patients compared to the overweight patients (Table 3).

\section{Discussion}

The greater the frequency of risk factors identified in mothers during pregnancy, the greater was the likelihood that the child's BMI-SDS would be increased (10). Mothers with a normal BMI usually gain 11 to $16 \mathrm{~kg}$ during pregnancy (11). In the present study we found in both countries that an excessive weight gain of mothers during pregnancy was associated with the risk for childhood obesity $(12,13)$.

The prevalence of birth before 37 or after 42 gestational weeks in the DE study population was within the expected range (14). These figures showed a somewhat higher prevalence in the UA group. Gestational hypertension was found to occur in 5-10\% of all pregnancies (15). The same prevalence was observed in the mothers of overweight children in our study. By contrast, the mothers of children with obesity were twice as likely to have developed gestational hypertension (17\%). However, this finding was limited to the DE population. There are no exact data on the prevalence of early contractions with estimates ranging from $5 \%$ up to $35 \%$ of pregnancies (16). If early contractions are considered as a potential threat of premature birth, the increased incidence may be considered as a relevant risk factor for childhood overweight and obesity. Perinatal asphyxia may be equally considered a risk factor. In our patients, the prevalence of perinatal asphyxia both in the UA and in the DE populations exceeded the prevalence of 0.5$1 \%$ (5-10:1000 births) observed in the general population (17).

Several studies have shown that the BMI-SDS of the parents plays an important role influencing the BMI-SDS of a child (18). In the current study, maternal BMI-SDS was significantly higher in obese children as compared to the values of mothers of overweight children. A highly significant positive correlation was found between the BMI-SDS of the children and the BMI-SDS of their parents. There was a significant positive correlation between the number of familial risk factors (diabetes mellitus and arterial hypertension) and the BMI-SDS values of the children .

The prevalence of arterial hypertension ranges from $32.3 \%$ in developed to $40.8 \%$ in developing countries (19). The present study indicates that first degree relatives of obese children have a higher prevalence of arterial hypertension and furthermore have a significantly higher prevalence of diabetes mellitus. According to the atlas of the International Diabetes Federation, the prevalence of diabetes in DE was $10.6 \%$ in 2015 , with a proportion of undetected diabetes of $38.2 \%$. In UA during the same year, the respective numbers were $8 \%$ and $43.2 \%$ (20). We suspect that in the UA population there may be a greater deficit in the diagnosis of DM and arterial hypertension in adults, and that the actual frequency is most likely significantly higher. This suspicion is supported by the increased incidence of obesity in families in the UA population.

Currently there are no studies showing the prevalence of obesity in one-year-old children. In the US, the prevalence among children aged 0-2 years was reported as high as $8.1 \%$ (21). In our study, the prevalence of overweight and obesity at the age of one year was much higher with $17-27 \%$ in the DE population and $14-44 \%$ for children in UA. The results support the potential importance of BMI in children under two years of age to identify an increased risk of later obesity (22). Furthermore, breastfeeding plays an important role in the prevention of obesity. Studies have shown that formulafed infants have a higher chance of becoming obese later in life compared to breastfed infants (23).

So far only a small number of studies have investigated the onset of obesity. Most of these studies have identified the preschool age of 5-7 years as a risk period (24). In our study, children and parents reported significant weight gain starting from the age of 5-6 years in the DE population and 7-10 years in the UA population. This information might be important to identify the right timing for intervention, investigation and prevention. The increase in BMI-SDS is influenced by the duration of overweight and/or obesity in childhood. Therefore, initiating intervention and therapy as early as possible is important. The children of the UA population had lower BMI-SDS compared to the DE population. To our knowledge this is the first study comparing these two countries.

The data on which parameter is the better one to describe abdominal fat distribution in children is controversial. While American sources showed that BMI and WHtR did not differ 
in identifying children with cardiovascular risk factors, other studies found that waist circumference and WHtR were better predictors of cardiovascular risks compared to BMI (25). Recent research has shown that WHR may not be an informative parameter for cardiometabolic risk. On the other hand, WHtR is associated with cardiometabolic risk compared to BMI-SDS in both adults and children. In both populations children showed trunkal obesity. In German children aged $12-18$ years WHR was reported as $0.83 \pm 0.05$ in boys and as $0.78 \pm 0.06$ in girls (26).

The normal value for $\mathrm{WHtR}$ has been reported to be below 0.5 (27). In our study, WHtR was $>0.5$ in children with obesity from the UA population and in children with overweight and with obesity from the DE population which may indirectly be interpreted as a cardiometabolic risk factor.

The prevalence of high blood pressure in obesity varies: from $21-35 \%$, up to $46 \%$ in children and $40 \%$ in adults (28). According to other studies the prevalence of hypertension in overweight children increased from $6.6 \%$ in boys aged 2-5 years to $13.3 \%$ in adolescents $16-19$ years; and in girls $4.4 \%$ and $16.3 \%$ respectively (29). In our study, we found a prevalence of $44.1 \%$ in the UA obese group while in the DE group the prevalence of hypertension was even higher, at $56.1 \%$.

Acanthosis nigricans is associated with diabetes mellitus type 2 and insulin resistance and correlates strongly with obesity, although it has been reported to be present in $17 \%$ of healthy children (30). Acanthosis nigricans was observed twice as often in the DE patients compared to the UA population with a moderately higher prevalence compared to healthy children. In both populations, acanthosis nigricans was significantly more common in obese patients than in patients who were overweight.

\section{Study Limitations}

The main limitation of our study is that we relied on selfreports of the parents for pregnancy history and history of weight gain of their children.

\section{Conclusion}

The anamnestic risk factors for overweight and obesity in children were very similar in the DE and UA subjects, except for the number of familial risk factors which did not correlate with the BMI-SDS values in the UA population. We assume that in the UA population there is a greater deficit in the diagnosis of diabetes mellitus and arterial hypertension in adults and that a substantial fraction of adult cases of diabetes and arterial hypertension remain undiagnosed in the UA. The actual prevalence of these conditions is likely to be significantly higher. Relevant risk factors for the development of obesity include family and pregnancy history, as well as neonatal and infant medical history. Further important risk factors include anthropometric parameters. Since BMI normal values are age-dependend and increase during adolescents, BMI-SDS should be used for the evaluation of the degree of childhood obesity. WHtR, blood pressure and presence of acanthosis nigricans are important prognostic indicators for the risk of obesity related diseases, and should be determined in children and adolescents with overweight/obesity. The children from the DE population became overweight significantly earlier compared to the UA population. DE patients with obesity also had higher BMI SDS.

\section{Ethics}

Ethics Committee Approval: The study was approved by the Ethics Committee of the Medical Faculty of the University of Heidelberg (approval number: S-337/2013, approval date: 22/07/2013).

Informed Consent: Written consent was taken from the parents at the beginning of the study in accordance with the Declaration of Helsinki.

Peer-review: Externally and internally peer-reviewed.

\section{Authorship Contributions}

Surgical and Medical Practices: Vira Yakovenko, Juergen Grulich-Henn, Concept: Juergen Grulich-Henn, Markus Bettendorf, Natalia Zelinska, Vira Yakovenko, Design: Juergen Grulich-Henn, Markus Bettendorf, Natalia Zelinska, Vira Yakovenko, Data Collection or Processing: Vira Yakovenko, Juergen Grulich-Henn, Galyna Soloviova, Analysis or Interpretation: Georg F. Hoffmann, Markus Bettendorf, Natalia Zelinska, Vira Yakovenko, Laura Henn, Literature Search: Vira Yakovenko, Galyna Soloviova, Writing: Vira Yakovenko, Juergen Grulich-Henn.

Financial Disclosure: The authors declared that this study received no financial support.

\section{References}

1. World Health Organization. Facts and figures on childhood obesity. Last accessed date: 13 October 2017 Available from: https://www.who. int/end-childhood-obesity/facts/en/

2. Blüher S, Meigen C, Gausche R, Keller E, Pfaffle R, Sabin M, Werther G, Odeh R, Kiess W. Age-specific stabilization in obesity prevalence in German children: a cross-sectional study from 1999 to 2008. Int J Pediatr Obes 2011;6:199-206. Epub 2010 Nov 23

3. Zelinska N, Yakovenko V. Children obesity in Ukraine. 91st Annual meeting ENDO 09. Washington D.C.: Meeting \& Exhibit Guide, Washington, 2009:1. 
4. Zelinska N, Rudenko N. Statistics of pediatric endocrinology in Ukraine: summary of 2016. Ukrainiane Journal of Pediatric Endocrinology 2017;2:5-17.

5. Adane AA, Mishra GD, Tooth LR. Maternal pre-pregnancy obesity and childhood physical and cognitive development of children: a systematic review. Int J Obes (Lond) 2016;40:1608-1618. Epub 2016 Aug 16

6. Lau EY, Liu J, Archer E, McDonald SM, Liu J. Maternal weight gain in pregnancy and risk of obesity among offspring: a systematic review. J Obes 2014;2014:524939. Epub 2014 Oct 2

7. Carolan-Olah M, Duarte-Gardea M, Lechuga J. A critical review: early life nutrition and prenatal programming for adult disease. J Clin Nurs 2015;24:3716-3729. Epub 2015 Aug 9

8. Vieira SA, Magalhaes TC, Ribeiro AQ, Priore SE, Franceschini Sdo $C$, Sant'Ana LF. Influence of weight gain rate on early life nutritional status and body composition of children. ScientificWorldjournal 2014;2014:616108. Epub 2014 Nov 4

9. Riedel C, von Kries R, Buyken AE, Diethelm K, Keil T, Grabenhenrich L, Müller MJ, Plachta-Danielzik S. Overweight in adolescence can be predicted at age 6 years: a CART analysis in German cohorts. PloS one 2014;9:93581.

10. Olstad DL, Teychenne M, Minaker LM, Taber DR, Raine KD, Nykiforuk $\mathrm{CI}$, Ball K. Can policy ameliorate socioeconomic inequities in obesity and obesity-related behaviours? A systematic review of the impact of universal policies on adults and children. Obes Rev 2016;17:11981217. Epub 2016 Aug 3

11. Rasmussen KM, Yaktine AL. Weight Gain During Pregnancy: Reexamining the Guidelines, 2009. Available from: https://www.ncbi. nlm.nih.gov/pubmed/20669500

12. Gaillard R, Felix JF, Duijts L, Jaddoe VW. Childhood consequences of maternal obesity and excessive weight gain during pregnancy. Acta Obstet Gynecol Scand 2014;93:1085-1089. Epub 2014 Oct 9

13. Santos S, Monnereau C, Felix JF, Duijts L, Gaillard R, Jaddoe VWV. Maternal body mass index, gestational weight gain, and childhood abdominal, pericardial, and liver fat assessed by magnetic resonance imaging. Int J Obes (Lond) 2019;43:581-593. Epub 2018 Sep 19

14. Weiss E, Krombholz K, Eichner M. Fetal mortality at and beyond term in singleton pregnancies in Baden-Wuerttemberg/Germany 20042009. Arch Gynecol Obstet 2014;289:79-84. Epub 2013 Jul 10

15. Lo JO, Mission JF, Caughey AB. Hypertensive disease of pregnancy and maternal mortality. Curr Opin Obstet Gynecol 2013;25:124-132.

16. Steer P. The epidemiology of preterm labour. BJOG 2005;112(Suppl 1): $1-3$.

17. McGuire W. Perinatal asphyxia. Clin Evid 2006:511-519.

18. Lim YM, Song S, Song WO. Prevalence and Determinants of Overweight and Obesity in Children and Adolescents from Migrant and Seasonal
Farmworker Families in the United States-A Systematic Review and Qualitative Assessment. Nutrients 2017:9.

19. Pereira M, Lunet N, Azevedo A, Barros H. Differences in prevalence, awareness, treatment and control of hypertension between developing and developed countries. J Hypertens 2009;27:963-975.

20. International Diabetes Federation. IDF diabetes atlas 8th edition ed, 2017.

21. Ogden CL, Carroll MD, Fryar CD, Flegal KM. Prevalence of Obesity Among Adults and Youth: United States, 2011-2014. NCHS data brief 2015:1-8.

22. Roy SM, Chesi A, Mentch F, Xiao R, Chiavacci R, Mitchell JA, Kelly A, Hakonarson H, Grant SF, Zemel BS, McCormack SE. Body mass index (BMI) trajectories in infancy differ by population ancestry and may presage disparities in early childhood obesity. J Clin Endocrinol Metab 2015;100:1551-1560. Epub 2015 Jan 30

23. Robinson S, Yardy K, Carter V. A narrative literature review of the development of obesity in infancy and childhood. J Child Health Care 2012;16:339-354. Epub 2012 Sep 14

24. Cheung PC, Cunningham SA, Naryan KMV, Kramer MR. Childhood Obesity Incidence in the United States: A Systematic Review. Child Obes 2016;12:1-11. Epub 2015 Nov 30

25. Freedman DS, Kahn HS, Mei Z, Grummer-Strawn LM, Dietz WH, Srinivasan SR, Berenson GS. Relation of body mass index and waistto-height ratio to cardiovascular disease risk factors in children and adolescents: the Bogalusa Heart Study. Am J Clin Nutr 2007;86:3340.

26. Haas GM, Liepold E, Schwandt P. Percentile curves for fat patterning in German adolescents. World J Pediatr 2011;7:16-23. Epub 2010 Dec 30

27. Lo K, Wong M, Khalechelvam P, Tam W. Waist-to-height ratio, body mass index and waist circumference for screening paediatric cardiometabolic risk factors: a meta-analysis. Obes Rev 2016;17:1258-1275. Epub 2016 Jul 25

28. Reinehr T, Holl RW, Wabitsch M. The German Working Group of Obesity in Childhood and Adolescence (AGA): improving the quality of care for overweight and obese children in Germany. Obes Facts 2008;1:26-32. Epub 2008 Feb 8

29. Falkner B, Gidding SS, Ramirez-Garnica G, Wiltrout SA, West D, Rappaport EB. The relationship of body mass index and blood pressure in primary care pediatric patients. J Pediatr 2006;148:195-200.

30. Kong AS, Williams RL, Rhyne R, Urias-Sandoval V, Cardinali G, Weller NF, Skipper B, Volk R, Daniels E, Parnes B, McPherson L; PRIME Net Clinicians. Acanthosis Nigricans: high prevalence and association with diabetes in a practice-based research network consortium-PRIME Net study. J Am Board Fam Med 2010;23:476-485. 